\title{
Stentless thoracic endovascular aortic repair of a chronic DeBakey IIIb aneurysm
}

\author{
Ahmed Sameh Eleshra, MD, ${ }^{\mathrm{a}, \mathrm{b}}$ Woon Heo, MD, ${ }^{\mathrm{a}}$ Kwang-Hun Lee, MD, PhD, ${ }^{\mathrm{c}}$ and \\ Suk-Won Song, MD, PhD ${ }^{\mathrm{a}}$, Seoul, Republic of Korea, and Mansoura, Egypt
}

\footnotetext{
From the Departments of ${ }^{a}$ Thoracic and Cardiovascular Surgery and ${ }^{c}$ Interventional Radiology, Gangnam Severance Hospital, Yonsei University College of Medicine, Seoul, Republic of Korea; and ${ }^{\mathrm{b}}$ Department of Vascular Surgery, Mansoura University Hospital, Mansoura University Faculty of Medicine, Mansoura, Egypt.

Disclosures: Authors have nothing to disclose with regard to commercial support.

Received for publication April 30, 2018; revisions received June 1, 2018; accepted for publication June 7, 2018; available ahead of print July 24, 2018.

Address for reprints: Suk-Won Song, MD, PhD, Department of Cardiovascular Surgery, Gangnam Severance Hospital, Yonsei University College of Medicine, 211 Eonju-ro, Gangnam-gu, Seoul 135-720, Korea (E-mail: sevraphd@yuhs.ac).

J Thorac Cardiovasc Surg 2019;157:e67-8

$0022-5223 / \$ 36.00$

Copyright (C) 2018 by The American Association for Thoracic Surgery

https://doi.org/10.1016/j.jtcvs.2018.06.025
}

$\checkmark$ Video clip is available online.

Thoracic endovascular aortic repair (TEVAR) for chronic DeBakey IIIb (CDIIIb) aneurysms remain controversial. ${ }^{1}$ Intimal tears below the celiac trunk, visceral branches, and intercostal arteries from the false lumen (FL), endoleaks, and stent-induced new entry tears remain as obstacles to favorable aortic remodeling and are limitations of TEVAR for CDIIIb aneurysms. ${ }^{1,2}$ We describe favorable results of stentless TEVAR for progressive dilatation of a (CDIIIb) aneurysm in a 44-year-old man with many reentry tears and communicating channels with small primary entry tears at the proximal descending thoracic aorta (DTA).

\section{CLINICAL SUMMARY}

A 44-year-old man had progressive dilatation of a CDIIIb aneurysm by more than $0.5 \mathrm{~cm}$ within the previous 6 months diagnosed on the basis of serial computed tomographic angiograms. The FL received blood flow through the entry tear at the proximal DTA, communicating channels (intercostal artery and lumbar artery), and large reentry tears of the visceral vessels (celiac artery and right renal artery) and the right external iliac artery.

We performed multimodality therapy closure of the entry tear and branch vessel reentry tear, in addition to embolization of the communicating channels, without an aortic true-lumen stent graft. The stentless TEVAR technique was performed as follows:

1. The aortogram confirmed the computed tomographic angiographic findings.

2. The primary entry tear was closed (Video 1) with a 12-mm diameter arterial vascular plug (AGA Medical Corp, Plymouth, Minn).

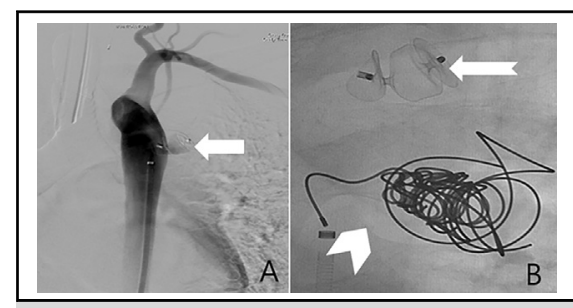

Stentless thoracic endovascular aortic repair with vascular plug (A) and coils (B).

Central Message

Stentless thoracic endovascular aortic repair is a safe procedure with favorable aortic remodeling.

See Commentary on page e69.

3. The FL of the DTA and 1 communicating channel of the left intercostal artery were closed with multiple 0.035-inch coils (Cook Medical LLC, Bloomington, Ind).

4. The celiac axis and the right renal artery reentry tears were closed with a covered stent $(9-\mathrm{mm} / 5-\mathrm{cm}$ and 7-mm/5-cm respectively, Viabahn; W.L. Gore and Associates, Flagstaff, Ariz).

5. The communicating channel of the L2 and L4 lumbar arteries were embolized with 0.035-inch coils (Cook Medical) and a 10-mm diameter arterial vascular plug (AGA Medical).

6. The right external iliac artery reentry tear was closed using a covered stent (11-mm/5-cm Viabahn; W.L. Gore and Associates).

The procedure was performed with the patient under general anesthesia without cerebral spinal fluid drainage.

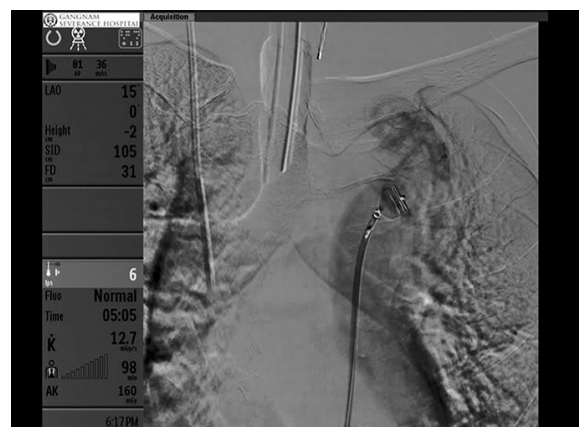

VIDEO 1. Angiogram of intimal tear localization and vascular plug insertion. Video available at: https://www.jtcvs.org/article/S0022-5223(18)31759-8/ fulltext. 
TABLE 1. Aortic diameter changes

\begin{tabular}{|c|c|c|c|c|c|c|c|c|c|}
\hline \multirow[b]{3}{*}{ Level } & & & & \multicolumn{6}{|c|}{ Follow-up CTA } \\
\hline & \multicolumn{3}{|c|}{ Preoperative CTA } & \multicolumn{3}{|c|}{$1 \mathrm{mo}$} & \multicolumn{3}{|c|}{9 mo } \\
\hline & TL & FL & Total & TL & FL & Total & TL & FL & Total \\
\hline LSA & 17.3 & 34.9 & 52.2 & 17.2 & 36.0 & 53.2 & 22.2 & 33.0 & 52.2 \\
\hline PAB & 12.8 & 33.7 & 46.5 & 15.7 & 31.7 & 47.4 & 22.1 & 24.7 & 46.8 \\
\hline $\mathrm{CA}$ & 14.0 & 20.9 & 34.9 & 13.5 & 22.0 & 35.5 & 20.0 & 14.0 & 34.0 \\
\hline
\end{tabular}

All data are in millimeters. $C T A$, Computed tomographic angiography; $T L$, true lumen; $F L$, false lumen; $L S A$, left subclavian artery; $P A B$, pulmonary artery bifurcation; $C A$, celiac axis.

This technique was approved by the institutional review board (Yonsei IRB no.3-2017-0359).

The patient's blood pressure had been well managed during the follow-up periods. We assessed aortic remodeling by changes of the aortic diameters at the left subclavian artery, the pulmonary artery bifurcation, and the celiac axis through computed tomographic angiography at 1, 6, and 9 months (Table 1). ${ }^{1}$ The overall diameter of the aorta was not decreased; however, the true lumen diameter increased at all levels after 9 months. In addition, disruption of the abdominal aortic flap occurred with patulous FL formation infrarenally with a 35-mm aortic diameter (Figure 1, $A-C$ ).

\section{DISCUSSION}

TEVAR for CDIIIb aneurysms remains controversial. ${ }^{1}$ Intimal tears below the celiac trunk, visceral branches, and intercostal arteries from the FL, endoleaks, and stent-induced new entry tears remain obstacles to favorable aortic remodeling and are limitations of TEVAR for CDIIIb aneurysms. ${ }^{1,2}$

We used FL procedures (arterial vascular plugs, coils, stents, and glues) after TEVAR for CDIIIb aneurysm as a means of promoting favorable aortic remodeling. Consequently, a complete thrombosis rate of $80 \%$ was observed.

When we revised our policy, we found that the main role of TEVAR was to seal a primary entry tear; otherwise, remodeling failed in more than $35 \%$ of our patients as a result of retrograde flow through visceral and iliac reentry sites in addition to intercostal and lumbar arteries, which require FL procedures. Why, therefore, do surgeons not embolize the FL by sealing proximal and distal entry tears with any communicating channel?

Stentless TEVAR is defined as an endovascular procedure with closure of the communicating channels between the true lumen and FL or obliteration of the FL itself both entry and re-entry tears using various materials. The suggested indication for our technique is chronic rather than acute dissection, especially for patients vulnerable to open repair with a primary tear smaller than 10 to $12 \mathrm{~mm}$ at the aortic arch or proximal DTA and those at risk for spinal cord ischemia with endograft coverage. In addition, the long duration of chronic dissection may hinder the expansion of the true lumen by the stent graft as a result of stiffness of the flap and may cause retrograde dissection, which is not a concern with our technique.

The technique described here is suitable for any CDIIIb aneurysm configuration, even in cases of compressed true lumen. In cases of severe compression, however, the true lumen may be occluded by a part of the vascular plug. In such cases, open surgical repair may be the treatment of choice.

We conclude that stentless TEVAR is a safe procedure that results in favorable aortic remodeling. It was especially favorable for this patient, who had many reentry tears and communicating channels with small primary entry tears at the proximal DTA.

\section{References}

1. Song SW, Kim TH, Lim SH, Lee KH, Yoo KJ, Cho BK. Prognostic factors for aorta remodeling after thoracic endovascular aortic repair of complicated chronic DeBakey IIIb aneurysms. J Thorac Cardiovasc Surg. 2014;148: 925-33.e1; discussion 932-3.

2. Idrees J, Roselli EE, Shafii S, Reside J, Lytle BW. Outcomes after false lumen embolization with covered stent devices in chronic dissection. J Vasc Surg. 2014;60:1507-13.

3. Kim TH, Song SW, Lee KH, Baek MY, Yoo KJ. Effects of false lumen procedures on aorta remodeling of chronic DeBakey IIIb aneurysm. J Ann Thorac Surg. 2016;102: 1941-7.

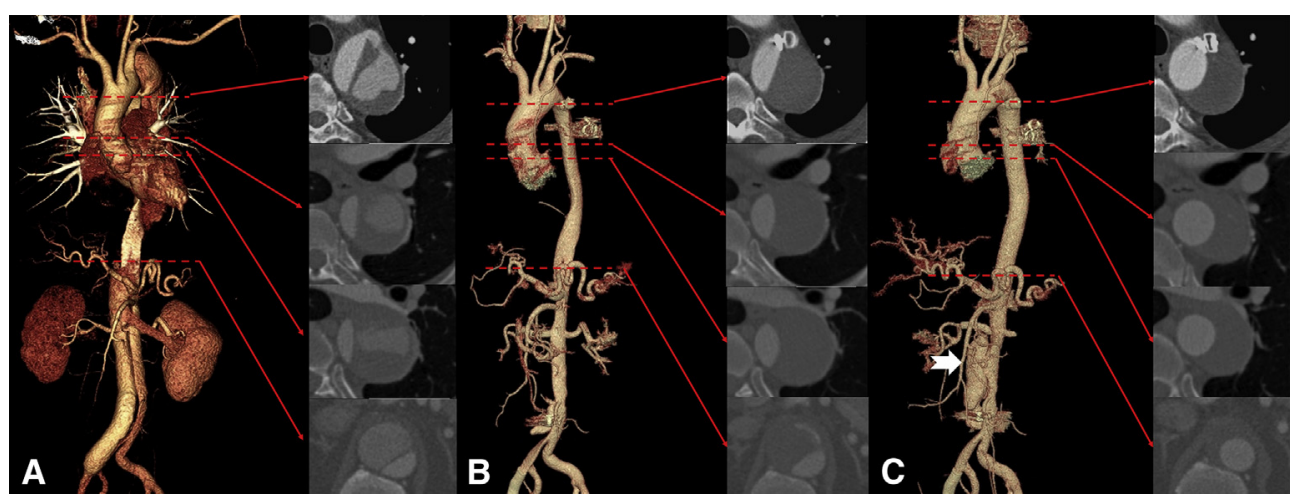

FIGURE 1. Three-dimensional reconstructions of the computed tomographic angiograms before the procedure (A), at 1 month after the procedure (B), and at 6 months after the procedure (C). Angiography demonstrates favorable aortic remodeling with an increase in the true lumen diameter (white arrow; disruption of the abdominal aortic flap with patulous false lumen formation infrarenally). Cross-sectional images of the indicated level (red dotted lines) are located as shown by the red arrows. 